\title{
Standpunkt
}

\section{Theorie und Praxis der anwaltsorientierten Juristenausbildung}

Was hat sich getan seit der Reform der Juristenausbildung?

\author{
Kristina Trierweiler"
}

Ziel des Gesetzes der Reform der Juristenausbildung, das am 1.7.2003 in Kraft trat, war u.a., der Berufspraxis in Universität und Referendariat mehr Geltung zu verschaffen. Insbesondere wurde Wert darauf gelegt, die Studierenden und Referendare auf den Beruf vorzubereiten, den sie am wahrscheinlichsten ergreifen werden, nämlich den des Rechtsanwalts. Die anwaltsorientierte Ausbildung sollte verstärkt werden.

Seitdem arbeiten die Universitäten daran, diese Vorgaben umzusetzen. Die Rechtsanwaltskammern tragen insbesondere in der Referendarausbildung ihren Teil zum Gelingen dazu bei, indem sie Arbeitsgemeinschaften anbieten, in denen materielles Recht aus anwaltlicher Perspektive und auch anwaltliches Berufsrecht gelehrt wird. Dennoch werden immer wieder Rufe laut, dass nicht alle Absolventen genügend auf die Tätigkeit des Anwalts vorbereitet sind. So fehle es an ausreichender Schriftsatztechnik oder rhetorischen Fähigkeiten. Demgegenüber wird von den Absolventen die berufliche Relevanz des Studiums nach einer Studie des International Center for Higher Education Research (INCHER) Kassel aus November 2013 rückblickend eher als gering angesehen. In den Jahrgängen 2008 bis 2011 haben durchschnittlich lediglich 36 Prozent der Studierenden an Lehrveranstaltungen mit spezifischem Bezug zum Anwaltsberuf teilgenommen. Was ist die Ursache? Ursache könnte die noch immer mangelnde Examensrelevanz von anwaltsspezifischen Themen sein. Das Interesse der Studierenden wird oftmals nur für prüfungsrelevanten Lernstoff geweckt. Anwaltsorientierte Themen werden noch immer in zu geringem Maße geprüft.

\section{A. Studium}

Die Universitäten müssen die Studierenden mit dem elementaren juristischen Handwerkszeug rüsten. Das Studium beinhaltet die rechtsprechende, verwaltende und rechtsberatende Praxis ( $\mathbb{S} 5 \mathrm{a} \mathrm{DRiG})$. Die anwaltliche Sichtweise ist dabei in die Lehrveranstaltungen einzubeziehen. Das Studium beinhaltet außerdem die Vermittlung von nichtjuristischen Fähigkeiten, und zwar Schlüsselqualifikationen wie Verhandlungsmanagement, Gesprächsführung, Rhetorik, Streitschlichtung, Mediation, Vernehmungslehre und Kommunikationsfähigkeit. Zudem haben die Studierenden während der vorlesungsfreien Zeit praktische Studienzeiten von mindestens drei Monaten $\mathrm{zu}$ absolvieren.

* Rechtsanwältin Kristina Trierweiler, LL.M. ist Mitglied der Geschäftsführung der Bundesrechtsanwaltskammer und dort u. a. zuständig für die Juristenausbildung. 
Die Rechtsanwaltskammern sind verpflichtet, bei der Ausbildung und Prüfung der Studierenden und Referendare mitzuwirken. Bis zum 30.6.2003 war die Pflicht der Kammern auf die Mitwirkung bei der Ausbildung beschränkt. Seit dem 1.7.2003 ist der Aufgabenbereich erweitert worden. Nunmehr hat der Kammervorstand bei der Ausbildung und Prüfung der Studierenden und der Referendare mitzuwirken, insbesondere qualifizierte Arbeitsgemeinschaftsleiter und Prüfer vorzuschlagen $(\mathbb{\$} 73$ Abs. 2 Nr. 9 BRAO). Darüber hinaus hat der Vorstand die anwaltlichen Mitglieder für die Prüfungsausschüsse der Ersten und Zweiten Juristischen Staatsprüfung vorzuschlagen ( $\$ 73$ Abs. 2 Nr. 10 BRAO). Die Einbeziehung der Anwaltschaft ist daher auf allen Ebenen im Rahmen von Vorlesungen, Arbeitsgemeinschaften, Klausurerstellung und in den juristischen Staatsprüfungen möglich. Die Rechtsanwaltskammern arbeiten mit den juristischen Fakultäten bei der inhaltlichen und methodischen Ausgestaltung des Anwaltsbezugs im Studium zusammen. Ausfluss dieser Neuerungen ist - und das liegt in der Natur der Sache -, dass die Fragen aus anwaltlicher Sicht zunehmen müssen, wenn die Prüfungsausschüsse regelmäßig auch mit Rechtsanwälten besetzt sind.

Um den besonderen Anforderungen im Examen gewappnet zu sein, ist es auch hilfreich, auf die Erfahrungen aus einem Praktikum in einer Rechtsanwaltskanzlei zurückgreifen zu können. An den Universitäten erfolgt die Wissensvermittlung in aller Regel durch Lösung von vorgegebenen Rechtsproblemen. Im Praktikum dagegen kann man lernen, aus einem umfassenden Lebenssachverhalt die relevanten Informationen herauszufiltern. Die Studierenden haben damit die Möglichkeit, einen ersten Einblick in die Praxis zu erhalten und sich frühzeitig beruflich zu orientieren. Wird das Praktikum ernst genommen, ist es mit einem effektiven Lerneffekt verbunden. Wenn der Praktikant in die Belange der Kanzlei eingebunden wird, er im Kanzleibetrieb mitwirken darf und sich der Ausbilder Zeit für die Betreuung seines Praktikanten nimmt, wird sich ein hoher Lernwert einstellen. Es besteht die Möglichkeit, Mandantengesprächen beizuwohnen und Gerichtsverhandlungen zu beobachten. Er wird mit wichtigen Fragen für den Anwaltsberuf konfrontiert, wie z.B. der Umgang mit den Mandanten, die Praxis der Verhandlungen bei Gericht, die Vorbereitung der Mandanten auf eine Gerichtsverhandlung, die Besprechung der Gebühren oder der Entscheidung, ob ein Mandat überhaupt angenommen werden soll. Der Studierende erfährt er, dass auch psychologische, kommunikative und kaufmännische Fähigkeiten für den Anwaltsberuf unerlässlich sind. Durch ein Praktikum werden auch die einfachen Dinge des Berufsalltags kennengelernt, wie Büroorganisation oder wie eine Akte aufgebaut ist. Nicht zuletzt geht es auch um die Erkenntnis, dass Rechtsanwälte unternehmerisch denken müssen. Schließlich ist nicht ausgeschlossen, dass sich im Anschluss die Gelegenheit ergibt, einen Job als studentische Hilfskraft zu ergattern.

Optimal wäre es, wenn - was leider nicht zwingend ist - das Praktikum in das Studium integriert würde, so wie es beispielsweise an der Rechtswissenschaftlichen Fakultät der Universität Köln praktiziert wird. Dort wird den Studierenden ein begleitendes Programm zur Vor- und Nachbereitung des Praktikums geboten. So werden 
die Studierenden in einem Workshop „Fit fürs Praktikum“ auf das Praktikum vorbereitet. U.a. erhalten sie eine Einführung in das anwaltliche Berufsrecht. Im Anschluss an das Praktikum besteht die Möglichkeit, durch das Verfassen eines Praktikumsberichts und dessen Vorstellung in einem Vortrag eine Schlüsselqualifikation zu erwerben.

Eine andere Möglichkeit, wie Theorie und Praxis wunderbar miteinander verzahnt werden können, sind Moot Courts, die sich immer größerer Beliebtheit erfreuen. Bei einem Moot Court wird eine Gerichtsverhandlung simuliert. So wurde am Institut für Prozess- und Anwaltsrecht der Juristischen Fakultät der Universität Hannover unter Beteiligung der Anwaltschaft in diesem Jahr zum zweiten Mal der Soldan Moot zur anwaltlichen Berufspraxis veranstaltet. Studierende werden auf diese Weise mit der Tätigkeit des Rechtsanwalts vertraut gemacht. Sie sollen als Interessenvertreter einen Fall rechtlich analysieren, Beweismittel würdigen und Rechtsmeinungen formulieren. Dabei müssen sie sich mit den Gegenargumenten auseinandersetzen und das Gericht von ihrer Position überzeugen. Die Teilnehmer lernen, Schriftsätze zu formulieren und Anträge zu stellen. Der Fall ist jeweils so gestaltet, dass den Studierenden auch wichtige Kenntnisse des anwaltlichen Berufsrechts vermittelt werden.

Auch sogenannte Law Clinics, die zunehmend Verbreitung finden, sind bestens geeignet, schon als Student mit den echten Fällen des Lebens vertraut zu werden und theoretisch erlangtes Wissen durch Praxiserfahrung anzureichern. Einer Law Clinic liegt das Konzept zu Grunde, dass Ratsuchende von Studierenden unentgeltlich beraten werden. Die Studierenden bearbeiten dabei unter Anleitung eines Volljuristen, z.B. eines Rechtsanwalts, geeignete Fälle mit geringem Streitwert. Die meist an den Juristischen Fakultäten angesiedelten Clinics haben sich auf die verschiedensten Rechtsgebiete spezialisiert, wie Grund- und Menschenrechte, Verbraucherrecht, Flüchtlingsrecht, Familienrecht, Sozialrecht u.v.m. Die Studierenden kommen bereits in einem frühen Stadium ihrer Ausbildung mit realen Mandanten, die aus den verschiedensten Gründen rechtliche Beratung bislang nicht in Anspruch genommen haben/nehmen konnten, in Kontakt. Sie erfahren außerdem, dass Jura mehr ist als trockene Theorie. Sie können sich in strategischem Denken, Gesprächsführung und Problembewusstsein üben. Es bietet sich an, die studentische Rechtsberatung als Schlüsselqualifikation anzuerkennen. An der Humboldt Universität zu Berlin zum Beispiel ist die Arbeit in den jeweiligen Clinics eng mit dem Lehrplan verknüpft, sodass sich praktische Erfahrungen und universitäre Lehre gegenseitig ergänzen.

An der Universität in Würzburg ist das Programm „Jura in der Praxis“ ins Leben gerufen worden. Das Programm kombiniert die Vorstellung der verschiedenen juristischen Berufsfelder durch Berufspraktiker mit Übungen, wie Rechtsberatung von Studierenden für Studierende, Moot Courts, Workshops etc. 


\section{B. Vorbereitungsdienst}

Die Reform der Juristenausbildung hat den Anwaltsbezug nicht nur im Studium, sondern auch im Vorbereitungsdienst gestärkt. Hiermit wurde einem langjährigen Petitum der Anwaltschaft entsprochen. Die Mindestdauer, die ein Referendar bei einem Rechtsanwalt in Ausbildung verbringt, beträgt neun Monate. Die Referendare können so intensiv in den Kanzleialltag eingebunden werden. Die Anwaltsstation als letzte Ausbildungsstage vor der Zweiten Juristischen Staatsprüfung wird aber gern als sogenannte Tauchstation benutzt. Dieses Phänomen besteht weiterhin fort. Auch wenn die Vorbereitungszeit auf die Examensprüfungen angesichts der Stofffülle von den Prüfungskandidaten oft als sehr knapp angesehen wird, kann nur dazu geraten werden, die Anwaltsstation auszuschöpfen. Wird man dort als vollwertige Arbeitskraft in den Kanzleialltag integriert, erschließt sich die anwaltliche Denk- und Arbeitsweise. Die Ausbildung am Arbeitsplatz bietet die Gelegenheit, die im Studium erlangten Kenntnisse in die Praxis umzusetzen und zu verfeinern. In einigen Bundesländern sind die Referendare verpflichtet, in der Rechtsanwaltsstation ein Berichtsheft zu führen. Dieses ist zu den Prüfungsakten zu reichen und soll helfen, einen Missbrauch der Anwaltsstation als Tauchstation zu bekämpfen.

In den Arbeitsgemeinschaften wird der Anwaltsbezug dadurch hergestellt, dass in vielen Bundesländern die Rechtsanwaltskammern Einführungslehrgänge veranstalten. Den Rechtsreferendaren werden auf diese Weise von Praktikern materielles Recht aus anwaltlicher Sicht sowie berufsrechtliche und berufspraktische Themen vermittelt.

Erfreulicherweise haben die Präsidenten der Landesjustizprüfungsämter beschlossen, dass seit Jahresbeginn im Zweiten Juristischen Staatsexamen eine der Klausuren zwingend eine zivilrechtliche rechtsgestaltende Anwaltsklausur sein muss. Für die Vorbereitung auf diese Art Klausuren ist es unerlässlich, tatsächlich in einer Kanzlei gearbeitet und den Blick durch die Anwaltsbrille geworfen zu haben. Insbesondere in der mündlichen Prüfung werden gern Fragen gestellt, deren Antworten keinem Lehrbuch entnommen werden können, sondern die nur aus der Erfahrung des anwaltlichen Tagesgeschäfts bekannt sein können.

\section{Fazit}

Festzustellen ist vorab, dass folgende Faktoren unsere juristische Ausbildung nach wie vor zu einem Qualitätssiegel machen: Zum einen die beiden Staatsprüfungen die Examenskandidaten müssen nicht nur Teilaspekte des Rechts kennen, um diese Prüfungen zu bestehen, es wird vielmehr gewährleistet, dass sie sowohl die Zusammenhänge zwischen den Rechtsgebieten als auch das juristische Grundsystem, das sie auf unbekannte Fälle anwenden müssen, verstanden haben. Zum anderen werden Richter, Rechtsanwälte und Staatsanwälte gemeinsam ausgebildet, sodass wir den Vorteil haben, uns in unserer Arbeit gegenseitig auf Augenhöhe begegnen zu können. Der Einheitsjurist ist hoch angesehen, im Ausland werden wir um ihn beneidet. 
Aber nicht nur auf die beiden Staatsprüfungen, sondern auch auf den Berufseinstieg müssen die Absolventen bestmöglich vorbereitet werden, damit sie auf dem Arbeitsmarkt erfolgreich sind und im Wettbewerb bestehen. Dazu ist es notwendig, dass sie für die wechselnden und vielschichtigen Anforderungen der Arbeitswelt qualifiziert werden. Informationen über die verschiedenen Aspekte des Berufs und die mannigfaltigen Anforderungen an den Beruf sind äußerst wichtig. Die juristische Ausbildung muss stets von dem Leitgedanken der Qualitätssicherung geprägt sein und sich Entwicklungen anpassen. Es ist unerlässlich, dass alle Akteure bereit sind, sich darauf einzulassen und intensiv mitzuwirken, wobei es in erster Linie jedem Absolventen selbst obliegt, das vielfältige Angebot in Studium und Referendariat zu nutzen und durch Eigeninitiative, Engagement, Neugier und stetige Lernbereitschaft den Grundstein für den beruflichen Erfolg zu legen.

Es scheint, dass sowohl im Studium als auch im Referendariat der rechtliche Rahmen für eine Verzahnung von Theorie und Praxis durchaus vorhanden ist. Es gilt, das traditionell theorielastige Studium mit Leben zu füllen. Viele sehr gute Ideen, von denen hier nur wenige benannt werden konnten, sind dazu bereits umgesetzt worden. Alle Bemühungen um einen Praxisbezug in der Lehre wären jedoch letztlich zum Scheitern verurteilt, wenn die anwaltsorientierte Sichtweise nicht auch in ausreichendem Maße Eingang in die zu fordernden Prüfungsleistungen fände. Es ist nun einmal so, dass die Prüfungskandidaten nur das lernen, was letztendlich auch geprüft wird. 